\title{
Non-Euclidean origami
}

\author{
Scott Waitukaitis, Peter Dieleman, and Martin van Hecke 10 \\ Huygens-Kamerlingh Onnes Laboratory, Leiden University, P.O. Box 9504, 2300 RA Leiden, The Netherlands \\ and AMOLF, Science Park 104, 1098 XG Amsterdam, The Netherlands
}

(Received 12 July 2019; accepted 31 August 2020; published 25 September 2020)

\begin{abstract}
Traditional origami starts from flat surfaces, leading to crease patterns consisting of Euclidean vertices. However, Euclidean vertices are limited in their folding motions, are degenerate, and suffer from misfolding. Here we show how non-Euclidean 4-vertices overcome these limitations by lifting this degeneracy, and that when the elasticity of the hinges is taken into account, non-Euclidean 4-vertices permit higher order multistability. We harness these advantages to design an origami inverter that does not suffer from misfolding and to physically realize a tristable vertex.
\end{abstract}

DOI: 10.1103/PhysRevE.102.031001

Origami provides a vast space to design novel mechanical metamaterials and folding devices [1-20]. The exceptional geometrical, shape-shifting, and mechanical functionalities of these systems ultimately spring from the nonlinear folding motions of the building blocks of origami [1-14,2030]. These building blocks are $n$-vertices-units where $n$ straight folds connected to $n$ rigid plates meet at a point $[1,2,4,5,17,18,20,28,31-33]$. Most attention has been on Euclidean vertices-which in their unfolded state lie flat in the plane-and in particular 4-vertices, as these have a single degree of freedom [Fig. 1(a)]. However, the folding motions of Euclidean 4-vertices are limited and degenerate. This degeneracy follows from fold-inversion symmetry: If the folded state of a vertex, specified by the folding angles $\left\{\rho_{i}\right\}$, constitutes a valid configuration, so does $\left\{-\rho_{i}\right\}$. Hence, the unfolded $\left\{\rho_{i}=0\right\}$ state of Euclidean 4-vertices is self-symmetric and nongeneric. This leads to a dual-branch structure, where two folding motions, I and II, intersect at the flat state $[17,22,28,34]$. In turn, this degeneracy makes Euclidean crease patterns prone to misfolding [34-36].

To lift this degeneracy, we consider non-Euclidean 4vertices [37], i.e., those where the sector angles, $\alpha_{i}$, add to $2 \pi+\varepsilon$ (Fig. 1). It is known that for $\varepsilon \neq 0$, the folding branches of 4-vertices split and recombine into new branches $[22,38]$, and that non-Euclidean vertices can form "bowls" or "cones" in the case of a negative angular surplus $(\varepsilon<$ $0)$, and "saddles" in the case of a positive angular surplus $(\varepsilon>0)$ —in contrast, Euclidean 4-vertices only admit "bird foot" mountain-valley (MV) patterns with one mountain and three valley folds (or vice versa, e.g., $\{ \pm \mp \pm \pm\}$ ) $[5,17,39-$ 41]. However, a complete picture of the folding motions of non-Euclidean vertices is missing, and their potential has been overlooked.

Here we show how non-Euclidean vertices enhance the functionality of origami-based devices and materials. First, we systematically evaluate the folding motions of non-Euclidean 4-vertices to show exactly how the branch splitting occurs and find that non-Euclidean 4-vertices feature two distinct types of nonmonotonic folding motions, in striking contrast to the monotonicity of the folding motions of Euclidean 4-vertices $[17,39]$. We then consider how the absence of misfoldings leads to more robust nonlinear mechanisms and leverage this to design an origami inverter. Finally, we show how branch splitting leads to a tuneable energy barrier which can be harnessed to control the stability landscape and physically realize a tristable vertex. Together, our work shows the versatility of non-Euclidean origami as building blocks for advanced mechanical metamaterials.

Branch splitting and folding curves. We start by determining the qualitative nature of the folding branches for generic vertices with $\varepsilon \neq 0$. For Euclidean vertices, the unique folds (the one with opposite folding angle from the others) follow from inequalities on the sector angles $\alpha_{i}[5,17]$, and we orient these vertices such that $\alpha_{2}>\alpha_{4}$ and folds 1 and 2 are the unique folds on branch I and II, respectively (see the Supplemental Material for details [42]). The non-Euclidean vertices we consider are nearly flat (small $|\varepsilon|$ ) and are derived from shrunken or expanded Euclidean ones. Maintaining our conventions during shrinkage-expansion, we isolate generic features even while working with specific examples.

We start by examining the branch splitting for a family of vertices with sector angles $\alpha_{i}=[1+$ $\varepsilon /(2 \pi)]\{\pi / 3, \pi / 2,3 \pi / 4,5 \pi / 12\}$. We numerically calculate the folding curves, $\rho_{i}\left(\rho_{j}\right)$, for $\varepsilon=0$ and several values of $\varepsilon \neq 0$ and plot these in Fig. 2 (for derivations of the folding curves, see, e.g., Refs. [16,17]). For $\varepsilon \neq 0$, distortion causes the Euclidean branches I and II to split into four disconnected branches that we label $A-D$ [Figs. 2(b)-2(d)]. For $\epsilon<0$, the vertex is either on branch $A$ or $C$, and for $\epsilon>0$ the vertex is on branch $B$ or $D$ (see the Supplemental Material [42]). Pairs of branches are related by the fold-inversion symmetry discussed above. Clearly, the folding motions of non-Euclidean 4-vertices do not have any branch points and are smooth. Assuming rigid folding, a non-Euclidean vertex's branch designation is fixed and cannot change (e.g., a rigid vertex on branch $A$ cannot switch to branch $C$ ). As a consequence, a non-Euclidean vertex is specified by both its sector angles and its branch label. 
(a)

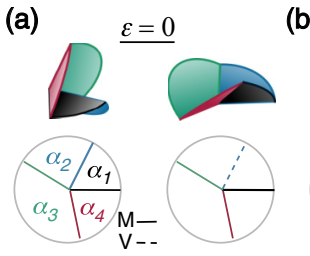

(b)

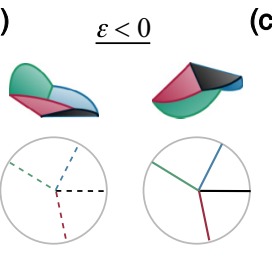

(c)

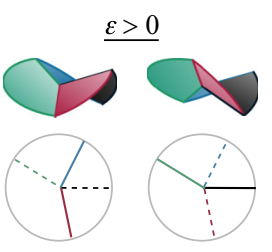

FIG. 1. (a) Folded states of an Euclidean 4-vertex (top) and corresponding MV patterns (bottom). (b) Non-Euclidean vertex with a negative angular surplus in a bowl or cone configuration. (c) Non-Euclidean vertex with a positive angular surplus in saddle configurations. In all cases, the given 4-vertex is specified by four sector angles, $\alpha_{i}$, and its folded states are described by four folding angles, $\rho_{i}$, which are the complements to the dihedral angles between plates $i$ and $i-1$.

Although corresponding to a specific choice of sector angles, the qualitative features of this splitting (up-down, right-left, four branches $A-D$ ) are completely general. To show this, and to connect the branches to qualitative shapescones, bowls, saddles, and bird's feet—we consider the sign of the folding angles along each branch, starting from their maximally folded states. We label the endpoints of the Euclidean folding branches as $\mathrm{I}^{+}:\{-+++\}, \mathrm{I}^{-}:\{+---\}, \mathrm{II}^{+}:\{+-$ $++\}$, and $\mathrm{II}^{-}:\{-+--\}$[Figs. 2(b)-2(d)]. For small $\varepsilon$, the non-Euclidean folding branches must have the endpoints close to their Euclidean parent, with the same MV patterns. The six possible folding branches of 4-vertices therefore follow from connecting pairs of endpoints. Euclidean folding branches I and II connect $\mathrm{I}^{+} \leftrightarrow \mathrm{I}^{-}$and $\mathrm{II}^{+} \leftrightarrow \mathrm{II}^{-}$, respectively, and intersect at the flat state. The remaining four endpoint-pair combinations correspond to the non-Euclidean folding branches $A-D$ that avoid the flat state (Fig. 2). We determine the MV patterns on each branch by noting that generically, one cannot have two fold angles pass through zero, or in other words, folds change between mountain and valley one by one along these branches. For example, on branch $A$, the endpoints $\{-+++\}$ and $\{+-++\}$ must be connected by the bowl $\{++++\}$, and therefore this branch must correspond to $\varepsilon<0$; similarly, on branch $B$, the endpoints $\{+-++\}$ and $\{+---\}$ must be connected by the saddle $\{+-+-\}$ and this branch must have $\varepsilon>0$; we summarize all six branches in Table 1 [43]. (Patterns with cyclic permutations of $\{++--\}$ are not allowed as they create intersections - see the Supplemental Material [42].)

Now that we have established how branches $A-D$ arise, we can determine how the folding motions along these branches differ qualitatively from those of Euclidean 4-vertices. For an

TABLE I. Folding branches and MV patterns.

\begin{tabular}{lccc}
\hline \hline & Endpoints & MV patterns \\
\hline $\mathrm{I}$ & $\mathrm{I}^{+} \leftrightarrow \mathrm{I}^{-}$ & $\{-+++\} \leftrightarrow\{0,0,0,0\} \leftrightarrow\{+---\}$ & $\varepsilon=0$ \\
$\mathrm{II}$ & $\mathrm{II}^{+} \leftrightarrow \mathrm{II}^{-}$ & $\{+-++\} \leftrightarrow\{0,0,0,0\} \leftrightarrow\{-+--\}$ & $\varepsilon=0$ \\
$A$ & $\mathrm{I}^{+} \leftrightarrow \mathrm{II}^{+}$ & $\{-+++\} \leftrightarrow\{++++\} \leftrightarrow\{+-++\}$ & $\varepsilon<0$ \\
$B$ & $\mathrm{II}^{+} \leftrightarrow \mathrm{I}^{-}$ & $\{+-++\} \leftrightarrow\{+-+-\} \leftrightarrow\{+---\}$ & $\varepsilon>0$ \\
$C$ & $\mathrm{I}^{-} \leftrightarrow \mathrm{II}^{-}$ & $\{+---\} \leftrightarrow\{----\} \leftrightarrow\{-+--\}$ & $\varepsilon<0$ \\
$D$ & $\mathrm{II}^{-} \leftrightarrow \mathrm{I}^{+}$ & $\{-+--\} \leftrightarrow\{-+-+\} \leftrightarrow\{-+++\}$ & $\varepsilon>0$ \\
\hline \hline
\end{tabular}

Euclidean vertex, the folding relations between any pair of $\rho_{i}$ on branches I or II are always monotonic and always capable of having positive or negative sign [17]. However, Table 1 and Fig. 2 show that for non-Euclidean vertices the qualitative nature of the folding motion depends on the branch and the pair of folding angles considered. First, monotonic folding curves with negative slope occur between the unique folds 1 and 2 for $\varepsilon<0$, or with positive slope between the nonunique folds 3 and 4 for $\varepsilon>0$ [Figs. 2(b) and 2(d)]. Between a unique $(1,2)$ and a nonunique $(3,4)$ fold, all curves are nonmonotonic. For $\varepsilon<0$, the unique fold monotonically changes sign, whereas the nonunique fold is nonmonotonic and has a fixed sign; for $\varepsilon>0$, the nonunique fold monotonically changes sign and the unique fold is nonmonotonic with fixed sign [see Fig. 2(c) for one such example]. Finally, between folds 1 and 2 for $\varepsilon>0$ and between the folds 3 and 4 for $\varepsilon<0$, both folds have a fixed sign and are nonmonotonic [Figs. 2(b) and 2(d)]. These qualitatively different folding motions open up new design possibilities for folding mechanisms, as well as rational design of multistable structures, as we show below.

Designer mechanisms.-We now show how the folding curves of a non-Euclidean vertex can be used to design nonlinear mechanisms. We illustrate this general point by designing an inverter, where a high input signal is mapped to a low output signal and vice versa [Fig. 3(a)]. In our origami inverter, the input and output signals correspond to fold angles. An Euclidean Miura vertex with sector angles $\alpha_{i}=\{\alpha, \pi-\alpha, \pi-\alpha, \alpha\}$ has one branch with a curve $\left(\rho_{i}\right.$ versus $\rho_{j}$ ) that reproduces a step function with infinite slope and sharp corners $[1,4,5,32,33,44]$ — close to the behavior desired for an inverter. However, the intersection of this branch at the flat state with the other distractor branch precludes the necessary one-to-one functionality.

To resolve this, we consider near-Miura, nonEuclidean candidates given by the sector angles $\alpha_{i}=$ $[1 \pm|\varepsilon| /(2 \pi)]\{\alpha-\delta, \pi-\alpha, \pi-\alpha+\delta, \alpha\}$. The parameter $\delta$ breaks the Miura symmetry, allowing us to (i) stay within our generic framework and (ii) control the sharpness of the step function. The general qualitative properties of the folding curves of non-Euclidean vertices (e.g., the curvature of the monotonic branch) prevent a single non-Euclidean vertex from achieving a folding branch with an $\mathrm{S}$ shape and inverter functionality. However, the absence of misfolding allows us connect multiple vertices without possible branch switching. By joining two vertices $\left(V_{1}\right.$ and $\left.V_{2}\right)$ and choosing their design and branches appropriately, we can achieve an origami inverter [Fig. 3(b)] as follows. Considering the input angle $\left(\rho_{\text {in }}^{1}\right)$, the connecting angle $\left(\rho_{\text {out }}^{1}=\rho_{\text {in }}^{2}\right)$, and the output angle $\left(\rho_{\text {out }}^{2}\right)$, the transfer curve is given by

$$
F\left(\rho_{\text {in }}^{1}\right)=\rho_{\text {out }}^{2}\left(\rho_{\text {out }}^{1}\left(\rho_{\text {in }}^{1}\right)\right) .
$$

The slope of a inverter curve must be negative, which accounting for the previously discussed (non-)monotonic nature of the non-Euclidean branches, is possible if for one vertex we use folds 1 and 2 and take $\epsilon>0$, and for the other we use folds 3 and 4 and take $\epsilon<0$. We therefore use as our input signal $\rho_{2}^{1}$ of vertex $V_{1}$ with $\alpha_{i}=[1-|\varepsilon| /(2 \pi)]\{\alpha-$ $\delta, \pi-\alpha, \pi-\alpha+\delta, \alpha\}$, and as our output signal $\rho_{3}^{2}$ of vertex $V_{2}$ with $\alpha_{i}=[1+|\varepsilon| /(2 \pi)]\{\alpha-\delta, \pi-\alpha, \pi-\alpha+\delta, \alpha\}$ 

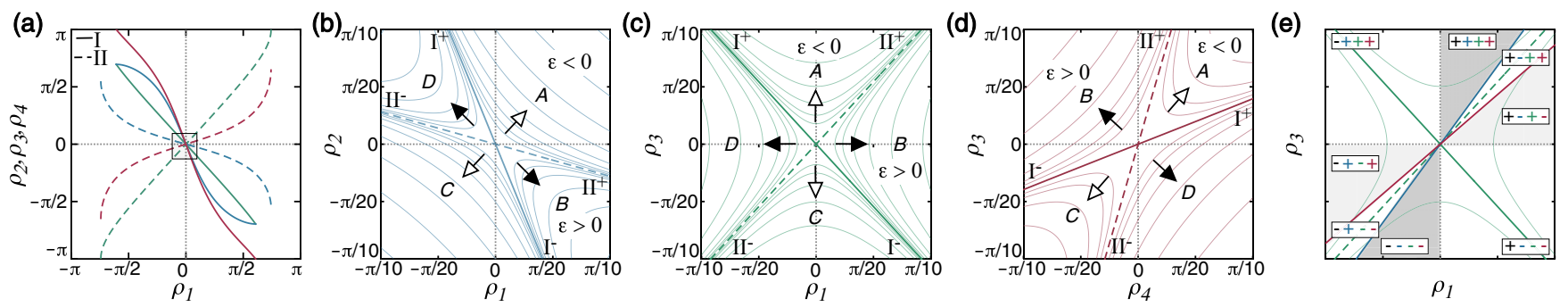

FIG. 2. (a) Folding curves $\rho_{2}$ (blue), $\rho_{3}$ (green), and $\rho_{4}$ (magenta) vs $\rho_{1}$ for branch I (solid) and II (dashed) of an Euclidean vertex with sector angles $\alpha_{i}=\{\pi / 3, \pi / 2,3 \pi / 4,5 \pi / 12\}$. The box in the center highlights enlarged views corresponding to panels (b)-(e), which also include curves for non-Euclidean vertices created by uniform shrinkage and expansion of $\alpha_{i}$. Open and closed arrows indicate splitting directions for $\varepsilon<0$ and $\varepsilon>0$, respectively. The branch endpoints $\mathrm{I}^{-}, \mathrm{I}^{+}, \mathrm{II}^{-}$, and $\mathrm{II}^{+}$are indicated, as well as the resulting non-Euclidean branch designations $A, B, C$, and $D$ (see Table I). (e) Schematic plot of $\rho_{3}$ vs $\rho_{1}$ with different MV assignments indicated with shading allows to visualize the sequence of MV patterns along each branch.

(connected by folds $\rho_{1}^{1}=\rho_{4}^{2}$ ). This yields the composite structure shown in Fig. 3(e), which produces a folding curve closely matching the target [Fig. 3(f); parameters in caption].

(a)

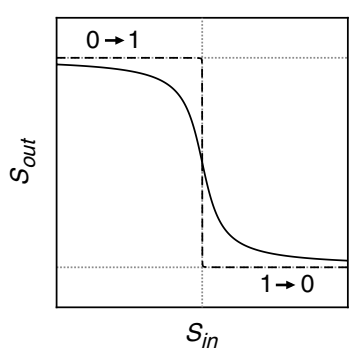

(c)

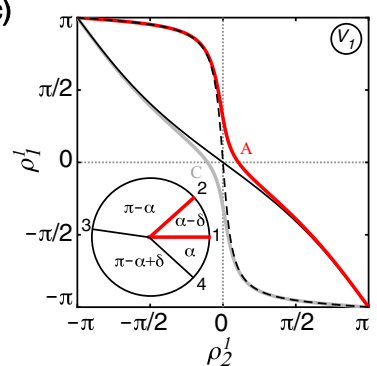

(e)

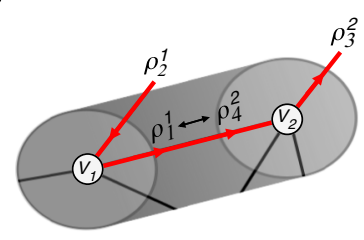

(b)

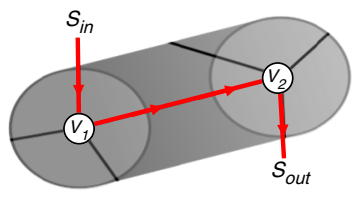

(d)

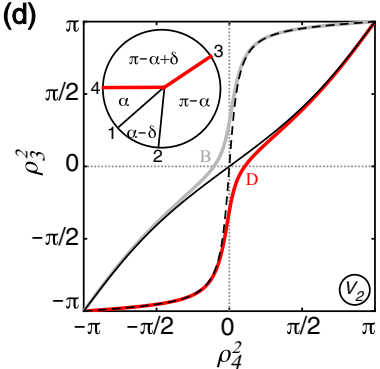

(f)

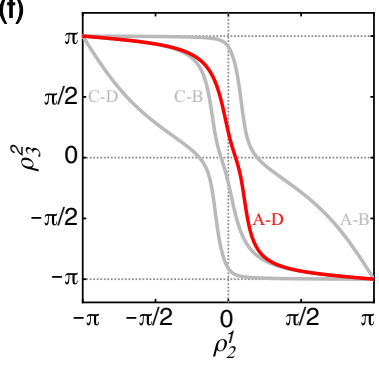

FIG. 3. (a) An inverter maps a high input signal, $S_{\text {in }} \approx 1$, to a low output signal, $S_{\text {out }} \approx 0$, and vice versa. (b) Composite design of two non-Euclidean 4-vertices, $V_{1}$ and $V_{2}$, with input-output corresponding to fold angles $S_{\text {in }}$ and $S_{\text {out }}$ (red). (c) Curve for vertex $V_{1}$ on branch $A$ (red); folding curves for the corresponding Euclidean vertex (black solid/dashed lines) and other branch of non-Euclidean vertex (gray) are also shown. (d) Curves for vertex $V_{2}$. (e) Origami inverter design. (f) Composite inverter curve (red) and spurious fold curves when one or both vertices are forced on their other branch (gray). Parameters for the target curve are $|\varepsilon|=\pi / 50, \alpha=\pi / 4$, and $\delta=0.1$.

Crucially, the other compound folding curves, obtained by changing the branch of $V_{1}, V_{2}$, or both, are well separated, even for small $(1 \%)$ deviation of flatness. This illustrates the potential of non-Euclidean origami for well-defined designer mechanisms that circumvent the problem of distractor branches.

Energy landscapes of non-Euclidean 4-vertices. The branch splitting has significant consequences for the energy and stability landscapes of non-Euclidean 4-vertices. When we model the hinge elasticity with torsional springs and assume the plates are rigid, the energy of a vertex is given by [3,4,17,20,20,21,45-47]:

$$
E=\frac{1}{2} \sum_{i=1}^{4} \kappa_{i}\left(\rho_{i}-\bar{\rho}_{i}\right)^{2} .
$$

Here $\kappa_{i}$ are the torsional spring constants and $\bar{\rho}_{i}$ are the rest angles of each fold. For an Euclidean vertex, the existence of two folding branches means that there are also two energy curves. These intersect at the flat state and, as we showed previously, this has the implication that generic Euclidean vertices are at least bistable. While more minima are possible - theoretically up to six [17] if there is sufficient freedom in the spring parameters - these populate a vanishingly small volume of design space and are too shallow to permit physical implementation [17].

To understand how non-Euclidean vertices differ, we consider what happens when a single torsional spring is placed on one of the folds. An Euclidean vertex will be able to reach a zero-energy minimum on both branches I and II, leading to two stable states. For a nearby non-Euclidean vertex, a different scenario emerges, and the nature of the folding motions suggests that the placement of the spring is critical. If $\epsilon<0$ and the spring is on a unique fold, then regardless of the branch the vertex is on ( $A$ or $C$ ) only one stable (zero-energy) minimum is accessible. (A similar case holds for $\epsilon>0$ with a spring on a nonunique fold.) However, if the spring is placed on a nonunique fold and $\varepsilon<0$, as in Fig. 4(a), then one branch ( $A$ if $\bar{\rho}>0$ ) will have two zero-energy minima, while the other will have a frustrated, finite-energy minimum near to the flat state. (Again, a similar situation happens for branches $B, D$ when $\epsilon>0$ and the spring is on a unique fold.) So long as the corresponding energy barrier can be exceeded, i.e., the plates are not too rigid [48] and can be bent or stretched, the vertex 
(a)

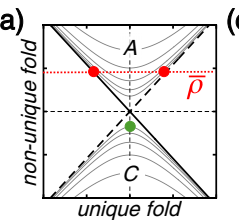

(c)
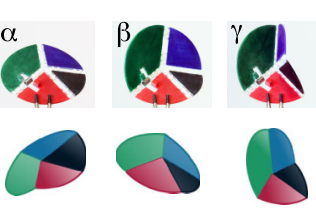

(b)
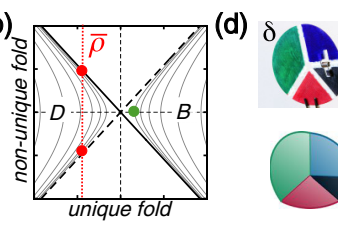

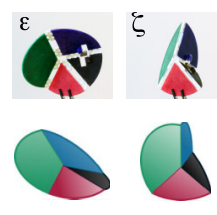

(e)
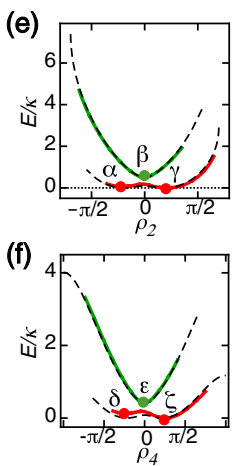

FIG. 4. (a) For a vertex with $\varepsilon<0$, placing a single spring on a nonunique fold with rest angle $\bar{\rho}>0$ (red line) yields two $E=0$ minima on the $A$ branch (red dots) and one $E \neq 0$ frustrated minimum on the $C$ branch (green dot). (b) Similar for a vertex with $\varepsilon>0$ and a spring placed on a unique fold. (c) Physical samples (top row) and numerical model (bottom row) for a 4-vertex $\alpha_{i}=$ $119 / 120\{\pi / 3, \pi / 2,3 \pi / 4,5 \pi / 12\}$ augmented by a torsional spring on fold 4 , in each of its three stable states labeled $\alpha-\gamma$. Plates are colored by the convention set in Fig. 1. (d) Physical samples (top row) and numerical model (bottom row) for a 4-vertex $\alpha_{i}=$ $121 / 120\{\pi / 3, \pi / 2,3 \pi / 4,5 \pi / 12\}$ augmented by a torsional spring on fold 2, in each of its three stable states labeled $\delta-\zeta$. [(e), (f)] By $3 \mathrm{D}$ printing vertices and adding springs, we are able to verify the effectiveness of these strategies. Panel (e) compares the theoretical (dashed lines) and experimentally measured (green and red lines) curves for the negative-surplus vertex. Panel (f) shows corresponding curves for a positive-surplus vertex. Both panels also indicate the $\operatorname{minima} \alpha-\zeta$.

can "pop through" the flat state [3]. This leads to a simple rule for creating robust tristable vertices: (1) for $\epsilon<0$, place a single spring on a nonunique fold; (2) for $\epsilon>0$, place a single spring on a unique fold.

For a physical realization, we 3D print nonEuclidean vertices with sector angles $\alpha_{i}=[1+$ $\varepsilon /(2 \pi)]\{\pi / 3, \pi / 2,3 \pi / 4,5 \pi / 12\}$ out of ABS plastic, which allows for a small amount of elastic deformation (for fabrication details, see the Supplemental Material [42]). With an appropriate value of angular surplus $(\varepsilon \approx \pm 0.0083)$, these vertices exhibit robust pop-through behavior. When additionally paired with a torsional spring-which can be mounted on the vertex in 3D printed holes-one of the two

branches can be made bistable, whereas the other branch is monostable with a frustrated minimum-validating the strategy for tristable vertices [Figs. 4(c) and 4(d)]. For both $\varepsilon>0$ and $\varepsilon<0$, we have measured the elastic energies along both branches and find that it compares well to the theoretical prediction based on the geometric design and torsional stiffness of our spring [Figs. 4(e) and 4(f)]. Hence, while the switching between branches relies on nonrigid deformations, the energetics along each branch are well described by a model based exactly on rigid origami. We therefore show that for non-Euclidean vertices with finite hinge and plate elasticity-the situation most relevant to many applications - independently tuning the energy barrier between branches and the energy landscape on the branches results in a strategy for multistable origami.

Conclusion and discussion.- Euclidean 4-vertices sit at a critical plane in parameter space and undergo a bifurcation when they are made non-Euclidean by shrinking or extending their sector angles. The associated branch splitting lifts the degeneracy in the folding motions, leads to new mountain valley patterns, and yields nonmonotonic folding curves. NonEuclidean 4-vertices do not suffer from distractor branches, and we have shown how to use this to design a nonlinear mechanism. Nonrigid, non-Euclidean 4-vertices can exhibit a pop through between branches, offering a simple pathway to tristable structures. While we have focused on single and dual non-Euclidean 4-vertices, we point out that a recent design methodology, initially developed for flat 4-vertices, can readily be adapted to design a wide variety of periodic and spatially textured crease patterns that combine non-Euclidean 4 -vertices with positive and negative angular surplus [14]. One interesting question for the future is how the nonmonotonic folding motions of individual 4-vertices affects those of larger folding patterns. A second question is how to extend our results to higher $n$ vertices, and in particular whether we can use higher $n$ vertices to design more complex mechanisms [49].

Acknowledgments. We thank C. Coulais, C. Santangelo, I. Cohen, and A. Evans for productive discussions, and M. Mertens for exploratory studies on the origami inverter. We acknowledge funding from the Netherlands Organization for Scientific Research through Grants VICI No. NWO-680-47609 (M.v.H. and S.W.) and VENI No. NWO-680-47-453 (S.W.).
[1] K. Miura, Method of packaging and deployment of large membranes in space, Inst. Space Astronaut. Sci. Rep. 618, 1 (1985).

[2] M. Schenk and S. D. Guest, Geometry of Miura-folded metamaterials, Proc. Nat. Acad. Sci. USA 110, 3276 (2013).

[3] J. L. Silverberg, A. A. Evans, L. McLeod, and R. C. Hayward, Using origami design principles to fold reprogrammable mechanical metamaterials, Science 345, 647 (2014).

[4] Z. Y. Wei, Z. V. Guo, L. Dudte, H. Y. Liang, and L. Mahadevan, Geometric Mechanics of Periodic Pleated Origami, Phys. Rev. Lett. 110, 215501 (2013).

[5] S. R. Waitukaitis and M. van Hecke, Origami building blocks: Generic and special four-vertices, Phys. Rev. E 93, 023003 (2016).
[6] K. C. Cheung, T. Tachi, S. Calisch, and K. Miura, Origami interleaved tube cellular materials, Smart Mater. Struct. 23, 094012 (2014).

[7] H. Yasuda and J. Yang, Reentrant Origami-Based Metamaterials with Negative Poisson's Ratio and Bistability, Phys. Rev. Lett. 114, 185502 (2015).

[8] B. G.-g. Chen, B. Liu, A. A. Evans, J. Paulose, I. Cohen, V. Vitelli, and C. D. Santangelo, Topological Mechanics of Origami and Kirigami, Phys. Rev. Lett. 116, 135501 (2016).

[9] L. H. Dudte, E. Vouga, T. Tachi, and L. Mahadevan, Programming curvature using origami tessellations, Nat. Mater. 15, 583 (2016). 
[10] J. T. B. Overvelde, T. A. de Jong, Y. Shevchenko, S. A. Becerra, G. M. Whitesides, J. C. Weaver, C. Hoberman, and K. Bertoldi, A three-dimensional actuated origami-inspired transformable metamaterial with multiple degrees of freedom, Nat. Commun. 7, 10929 (2016).

[11] J. T. B. Overvelde, J. C. Weaver, C. Hoberman, and K. Bertoldi, Rational design of reconfigurable prismatic architected materials, Nature (London) 541, 347 (2017).

[12] E. Boatti, N. Vasios, and K. Bertoldi, Origami metamaterials for tunable thermal expansion, Adv. Mater. 29, 1700360 (2017).

[13] K. Bertoldi, V. Vitelli, J. Christensen, and M. V. Hecke, Flexible mechanical metamaterials, Nat. Rev. Mater. 2, 17066 (2017).

[14] P. Dieleman, N. Vasmel, S. Waitukaitis, and M.V. Hecke, Jigsaw puzzle design of pluripotent origami, Nat. Phys. 16, 63 (2020).

[15] Z. Wang, L. Jing, K. Yao, Y. Yang, B. Zheng, C. M. Soukoulis, H. Chen, and Y. Liu, Origami-based reconfigurable metamaterials for tunable chirality, Adv. Mater. 29, 1700412 (2017).

[16] A. A. Evans, J. L. Silverberg, and C. D. Santangelo, Lattice mechanics of origami tessellations, Phys. Rev. E 92, 013205 (2015).

[17] S. R. Waitukaitis, R. Menaut, B. G.-g. Chen, and M. van Hecke, Origami Multistabilty: From Single Vertices to Metasheets, Phys. Rev. Lett. 114, 055503 (2015).

[18] H. Fang, S. Li, H. Ji, and K. W. Wang, Uncovering the deformation mechanisms of origami metamaterials by introducing generic degree-four vertices, Phys. Rev. E 94, 043002 (2016).

[19] E. T. Filipov, T. Tachi, and G. H. Paulino, Origami tubes assembled into stiff, yet reconfigurable structures and metamaterials, Proc. Natl. Acad. Sci. USA 112, 12321 (2015).

[20] V. Brunck, F. Lechenault, A. Reid, and M. Adda-Bedia, Elastic theory of origami-based metamaterials, Phys. Rev. E 93, 033005 (2016).

[21] J. L. Silverberg, J.-H. Na, A. A. Evans, B. Liu, T. C. Hull, C. D. Santangelo, R. J. Lang, R. C. Hayward, and I. Cohen, Origami structures with a critical transition to bistability arising from hidden degrees of freedom, Nat. Mater. 14, 389 (2015).

[22] C. D. Santangelo, Extreme mechanics: Self-folding origami, Annu. Rev. Condens. Matter Phys. 8, 165 (2017).

[23] B. Liu, J. L. Silverberg, A. A. Evans, C. D. Santangelo, R. J. Lang, T. C. Hull, and I. Cohen, Topological kinematics of origami metamaterials, Nat. Phys. 14, 811 (2018).

[24] H. Yasuda, T. Tachi, M. Lee, and J. Yang, Topological kinematics of origami metamaterials, Nat. Commun. 8, 962 (2017)

[25] L. L. Howell, R. J. Lang, M. Frecker, and R. J. Wood, Special issue: Folding-based mechanisms and robotics, J. Mech. Robot 8, 030301 (2016).

[26] J. Morgan, S. P. Magelby and L. L. Howell, An approach to designing origami-adapted aerospace mechanisms, J. Mech. Design 138, 052301 (2016).

[27] S. H. Chen and L. Mahadevan, Rigidity percolation and geometric information in floppy origami, Proc. Natl. Acad. Sci. USA 116, 8119 (2019).

[28] B. G-g. Chen and C. D. Santangelo, Branches of Triangulated Origami Near the Unfolded State, Phys. Rev. X 8, 011034 (2018).

[29] N. P. Bende, A. A. Evans, S. Innes-Gold, L. A. Martin, I. Cohen, R. C. Hayward, and C. D. Santangelo, Geometrically controlled snapping transitions in shells with curved creases, Proc. Natl. Acad. Sci. USA 112, 11175 (2015).
[30] S. R. Waitukaitis, Clicks for doughnuts, Nat. Phys. 14, 777 (2018).

[31] B. H. Hanna, J. M. Lund, R. J. Lang, S. P. Magleby, and L. L. Howell, Waterbomb base: A symmetric single-vertex bistable origami mechanism, Smart Mater. Struct. 29, 094009 (2014).

[32] P. Sareh and S. D. Guest, Design of isomorphic symmetric descendants of the Miura-ori, Smart Mater. Struct. 24, 085001 (2015)

[33] P. Sareh and S. D. Guest, Design of non-isomorphic symmetric descendants of the Miura-ori, Smart Mater. Struct. 24, 085002 (2015).

[34] M. Stern, M. B. Pinson, and A. Murugan, The Complexity of Folding Self-Folding Origami, Phys. Rev. X 7, 041070 (2017).

[35] T. Tachi and T. C. Hull, Self-foldability of rigid origami, J. Mech. Robot 9, 021008 (2017).

[36] M. Stern, V. Jayaram, and A. Murugan, Shaping the topology of folding pathways in mechanical systems, Nat. Commun. 9, 4303 (2018).

[37] The plates and folds in the origami that we consider here are flat and straight, and individual vertices are polyhedral. However, the Gaussian curvature is clearly singular at each vertex center, and larger fold patterns form what can naturally be seen as discretized non-Euclidean surfaces.

[38] M. Berry, M. E. Lee-Trimble, and C. D. Santangelo, Topological transitions in the configuration space of non-Euclidean origami, Phys. Rev. E 101, 043003 (2020).

[39] D. A. Huffman, Curvature and creases: A primer on paper, IEEE Trans. Electron. Comput. C-25, 1010 (1976).

[40] We routinely denote MV patterns by the four signs of $\rho_{i}$ (e.g., $\{+,-,-,-\})$, where mountains are negative.

[41] Z. Abel, J. Cantarella, E. D. Demaine, D. Eppstien, T. C. Hull, J. S. Ku, R. J. Lang, and T. Tachi, Rigid origami vertices: Conditions and forcing sets, J. Comp. Geom. 7, 171 (2016).

[42] See Supplemental Material at http://link.aps.org/supplemental/ 10.1103/PhysRevE.102.031001 for additional details on the analysis and experiments. The Supplemental Material also makes references to Refs. [17,5,39].

[43] We have tacitly assumed a specific ordering of $\mathrm{I}^{+}$and $\mathrm{II}^{-}$in, e.g., in the $\rho_{1}-\rho_{2}$ plane. Reversing this order is tantamount to reversing I and II, which reverses $A \leftrightarrow C$ and $B \leftrightarrow D$, but which does not change the conclusions regarding the sequences of $\mathrm{MV}$ patterns for $\varepsilon<0$ and $\varepsilon>0$.

[44] A. Papa and S. Pellegrino, Systematically creased thin-film membrane structures, J. Spacecraft Rockets 45, 10 (2008).

[45] F. Lechenault, B. Thiria, and M. Adda-Bedia, Mechanical Response of a Creased Sheet, Phys. Rev. Lett. 112, 244301 (2014).

[46] T. Joules, F. Lechenault, and M. Adda-Bedia, Local mechanical description of an elastic fold, Soft Matter 15, 1619 (2019).

[47] E. T. Filipov, K. Liu, T. Tachi, M. Schenk, and G. H. Paulino, Bar and hinge models for scalable analysis of origami, Internat. J. Solids Structs. 124, 26 (2017).

[48] K. Liu and G. H. Paulino, Nonlinear mechanics of non-rigid origami: An efficient computational approach, Proc. R. Soc. London A 473, 20170348 (2017).

[49] Y. P. Song, R. M. Panas, S. Chizari, L. A. Shaw, J. A. Jackson, J. B. Hopkins, and A. J. Pascall, Additively manufacturable micro-mechanical logic gates, Nat. Commun. 10, 882 (2019). 